
\title{
25 Research Soure \\ Utilization of chronic lung disease treatment before the respiratory syncytial virus season
}

\section{Yoonyoung Choi ( $\nabla$ chyoonyo@ufl.edu )}

Merck: Merck \&amp; Co Inc https://orcid.org/0000-0002-8810-7579

\section{H. Cody Meissner}

Tufts University School of Medicine

\section{Christian Hampp}

US Food and Drug Administration

Haesuk Park

University of Florida

Almut G. Winterstein

University of Florida

\section{Short Report}

Keywords: chronic lung disease, treatment utilization pattern, respiratory syncytial virus, and palivizumab immuunoprophylaxis

Posted Date: March 29th, 2021

DOI: https://doi.org/10.21203/rs.3.rs-306149/v1

License: (c) (i) This work is licensed under a Creative Commons Attribution 4.0 International License.

Read Full License

Version of Record: A version of this preprint was published at European Journal of Pediatrics on August 7th, 2021. See the published version at https://doi.org/10.1007/s00431-021-04233-6. 


\section{Abstract}

Guidelines recommend palivizumab immunoprophylaxis for children with CLD in their second year of life if they continue to need treatment within 6 months before the RSV season. The utilization patterns of treatment (chronic corticosteroid therapy, diuretic therapy, or supplemental oxygen) are not well understood. We examined variations in CLD treatment for ten consecutive 20-day segments preceding RSV season onset. Among infants and children with CLD $(n=19,026), 35.2 \%$ received one or more medical treatments for CLD any time within 200 days before entering the second RSV season: $8.6 \%, 3.2 \%$, and $29.7 \%$ received oxygen, diuretics, and corticosteroids, respectively. Utilization decreased as infants' age increased with corticosteroids surpassing oxygen and diuretics. To avoid the capture of intermittent use of corticosteroids for acute infections, we found a minimum of 45 days cumulative exposure was necessary to determine chronic use.

\section{Introduction}

Guidelines from the American Academy of Pediatrics recommend palivizumab immunoprophylaxis for children with chronic lung disease of prematurity (CLD) in the second year of life if they require medical treatment (chronic corticosteroid therapy, diuretic therapy, supplemental oxygen) within six months before the onset of the respiratory syncytial virus (RSV) season. ${ }^{1}$ This requirement is used as a proxy for disease severity in an attempt to identify children with increased risk for RSV infection requiring hospitalization and thus with the greatest benefit from immunoprophylaxis. Limited information is available regarding the frequency and duration of such medical treatment in this clinical scenario. Thus, to inform clinical decision-making, we examined the utilization of medical treatment in this cohort.

\section{Methods}

We conducted a retrospective cohort analysis using a unique linkage of the Medicaid Analytic eXtract (MAX) databases (1999-2010) and Birth Certificate data from Florida and Texas. ${ }^{2,3}$ Children with records in both datasets had at least one medical encounter with a diagnosis of CLD (International Classification of Diseases, 9th revision, Clinical Modification code [ICD9-CM] 770.7: chronic respiratory disease arising in the perinatal period, or 769: respiratory distress syndrome (RDS) in newborn, which has been previously validated) ${ }^{4}$; who were according to their vital record born at $<32$ weeks of gestation; had $\geq 200$ days of continuous enrollment in Medicaid and were $\leq 24$ months of age at the start of the RSV season; and had received supplemental oxygen or mechanical ventilation during the first 90 days after birth (as ascertained from vital records and claims data). Details of operational definitions have been published in a previous study. ${ }^{5}$

We defined ongoing medical treatment of CLD as the administration of diuretic therapy, supplemental oxygen, or chronic corticosteroid therapy. 
Diuretic and corticosteroid therapies were ascertained from pharmacy claims using AHFS class 40:28 diuretics and AHFS 24:32.20 mineralocorticoid (aldosterone) receptor antagonists; and 68:04 adrenals, respectively. We calculated the duration of treatment based on pharmacy-entered days' supply (for corticosteroids and diuretics), assuming that treatment would start on the day of dispensing. Oxygen supplementation or ventilation was ascertained from medical claims using Healthcare Common Procedure Coding System codes (E0424, E0425, E0430, E0431, E0433, E0434, E0435, E0439, E0440, E0441, E0442, E0443, E0444, E0450, E1390, E1391, E1392, E1400, E1401, E1402, E1403, E1404, E1405, E1406), and ICD-9-CM diagnosis (V462) and procedure (93.96) codes, and from documentation of the need for assisted ventilation on birth certificates. We calculated the duration of oxygen supply based on recorded length of stay (for inpatient claims) and commonly observed intervals between claims (for outpatient claims, 30 days or 1 day when attributed to procedures or diagnoses, respectively).

To operationalize "chronic" corticosteroid therapy, we reviewed dispensing patterns among children with CLD diagnoses during 0-24 months of age. Most corticosteroid refills (81\%) occurred within 90 days of the previous fill, included both inhaled and oral dosage forms and inhaled dosage forms were commonly dispensed for a long days' supply. Accordingly, we calculated cumulative days' supply including both oral and inhaled corticosteroids by looking back 90 days from an index corticosteroid dispensing. For example, for a corticosteroid fill on March 31st, 2010, every corticosteroid fill between January 1st and March 31st would contribute to determining the number of cumulative days' supply during the 90-days look-back period. Each corticosteroid dispensing served as an anchor to calculate previous corticosteroid exposure. We used four thresholds to define "chronic" corticosteroid therapy: any corticosteroid dispensing, or $\geq 30-, 45$, or 60 -day cumulative days' supply during the past 90 days.

We also examined the concurrence of RTI infections to help to differentiate intermittent versus chronic corticosteroid therapy.

Utilization prevalences were calculated in 20-day segments across 200 days before the onset of the RSV season. As done in a previous study ${ }^{5}$, we defined RSV season onset as the first of the 26 consecutive weeks with the highest incidence of RSV hospitalizations in Florida and Texas, accounting for palivizumab exposure. We further stratified utilization patterns for infants and children by chronologic age: age 200-365 days and age 366-730 days at the start of the RSV season.

This study was approved by the institutional review and privacy boards of the University of Florida, the Centers for Medicare and Medicaid Services, and the Florida and Texas Departments of Health.

\section{Results}

Among infants and children with CLD $(n=19,026), 35.2 \%$ received one or more ongoing medical treatments for CLD any time within 200 days before entering their second RSV season: $8.6 \%, 3.2 \%$, and $29.7 \%$ received oxygen, diuretics, and corticosteroids, respectively. Among children aged 200-365 days at the start of the RSV season, the most distant time of the 200 days look-back period relied on oxygen and 
to a lesser extent, diuretic therapy (Fig. 1). In the second year of life, chronic corticosteroid use surpassed both diuretics and oxygen.

Utilization patterns of oxygen and diuretics within 200 days prior to the RSV season were not affected by RTI trends (Fig. 1). Prevalences of oxygen and diuretics use decreased as infants were getting older, approaching the start of the season. In contrast, corticosteroid dispensing showed a bimodal distribution with an unexpected increase at the beginning of the season (i.e., when children had the highest age and would be expected to have decreased CLD severity).

The increase was consistent with RTI incidence patterns in the study populations: the 200-day look-back period overlapped with an offset of the previous cold season and an onset of the current cold season, likely creating a composite of corticosteroid therapy that was used for seasonal acute respiratory tract infections as well as unresolved CLD. To associate corticosteroid use with the underlying CLD severity, rather than a seasonal respiratory infection, we compared trends of our various definitions of chronic corticosteroid based on cumulative days' supply during the past 90 days. As a result, shorter courses of chronic corticosteroid use paralleled the increasing RTI infection rates at RSV season onset, but this effect was attenuated when requiring $\geq 45$ cumulative days' supply to define chronic steroid use.

\section{Discussion}

We described patterns of medical treatment among children diagnosed with CLD who are entering their second RSV season. We observed decreasing utilization trends in oxygen and diuretics, as expected with increasing age. In the absence of a widely acknowledged definition of chronic corticosteroid therapy in this setting, our multiple threshold analyses elucidated the heterogeneous purpose of corticosteroids use, given their role in inflammation reduction. ${ }^{6}$ Our analysis suggests that the use of a more restrictive threshold (e.g. >45-day cumulative supply within the past 90 days) for corticosteroid prescriptions would more accurately capture chronic use to fulfill criteria for immunoprophylaxis.

A few limitations of this study are noteworthy. While Medicaid billing records are a reliable source for ascertaining medical support for CLD, ${ }^{7}$ claims-based treatment duration information can be inaccurate. In addition, our CLD cohort may not exactly mirror those recommended for palivizumab immunoprophylaxis in guidelines. For example, our data could not provide the actual duration of oxygen use that guidelines require to determine the eligibility (i.e., supplemental oxygen at least 28 days after birth). Finally, our operational definition for chronic corticosteroids use included inhaled corticosteroids based on an empirical finding of its prevalent use. Given a continuous evaluation of the beneficial effects of inhaled corticosteroid on the pulmonary system ${ }^{8}$, we acknowledge a restriction to systemic corticosteroids, which were not investigated in this study on their own, could have been relevant.

Our findings on the utilization of CLD treatment could inform guidelines to specify treatment requirements as a proxy for CLD severity (e.g., supplemental oxygen, diuretics, or $\geq 45$ cumulative day's 
supply of corticosteroids) and clarify the size of the eligible population for palivizumab prophylaxis among those in the second year of life.

\section{References}

1. Committee on Infectious Diseases (2014) Updated guidance for palivizumab prophylaxis among infants and young children at increased risk of hospitalization for respiratory syncytial virus infection. Pediatrics 134(2):e620-e638

2. Florida Department of Health. Certificates-birth, death, fetal death, marriage and divorce. http://www.floridahealth.gov/certificates /certificates/. Accessed July 1, 2015

3. Texas Health and Human Services. Texas vital statistics. https://www.dshs.texas.gov/vs/. Accessed July 1, 2015

4. Landry JS, Croitoru D, Menzies D (2012) Validation of ICD-9 diagnostic codes for bronchopulmonary dysplasia in Quebec's provincial health care databases. Chronic Dis Inj Can 33(1):47-52

5. Choi Y, Meissner HC, Hampp C, Park H, Brumback B, Winterstein AG (2020) Calibration of Chronic Lung Disease Severity as a Risk Factor for Respiratory Syncytial Virus Hospitalization. Journal of the Pediatric Infectious Diseases Society. Sep 26

6. Baveja R, Christou H (2006) Pharmacological strategies in the prevention and management of bronchopulmonary dysplasia. Semin Perinatol Aug 30(4):209-218

7. Crystal S, Akincigil A, Bilder S, Walkup JT (2007) Studying prescription drug use and outcomes with medicaid claims data: strengths, limitations, and strategies. Med Care Oct 45(10 Supl 2):S58-S65

8. Shinwell ES, Portnov I, Meerpohl JJ, Karen T, Bassler D. Inhaled corticosteroids for bronchopulmonary dysplasia: a meta-analysis. Pediatrics. 2016 Dec 1;138(6):e20162511

\section{Figures}




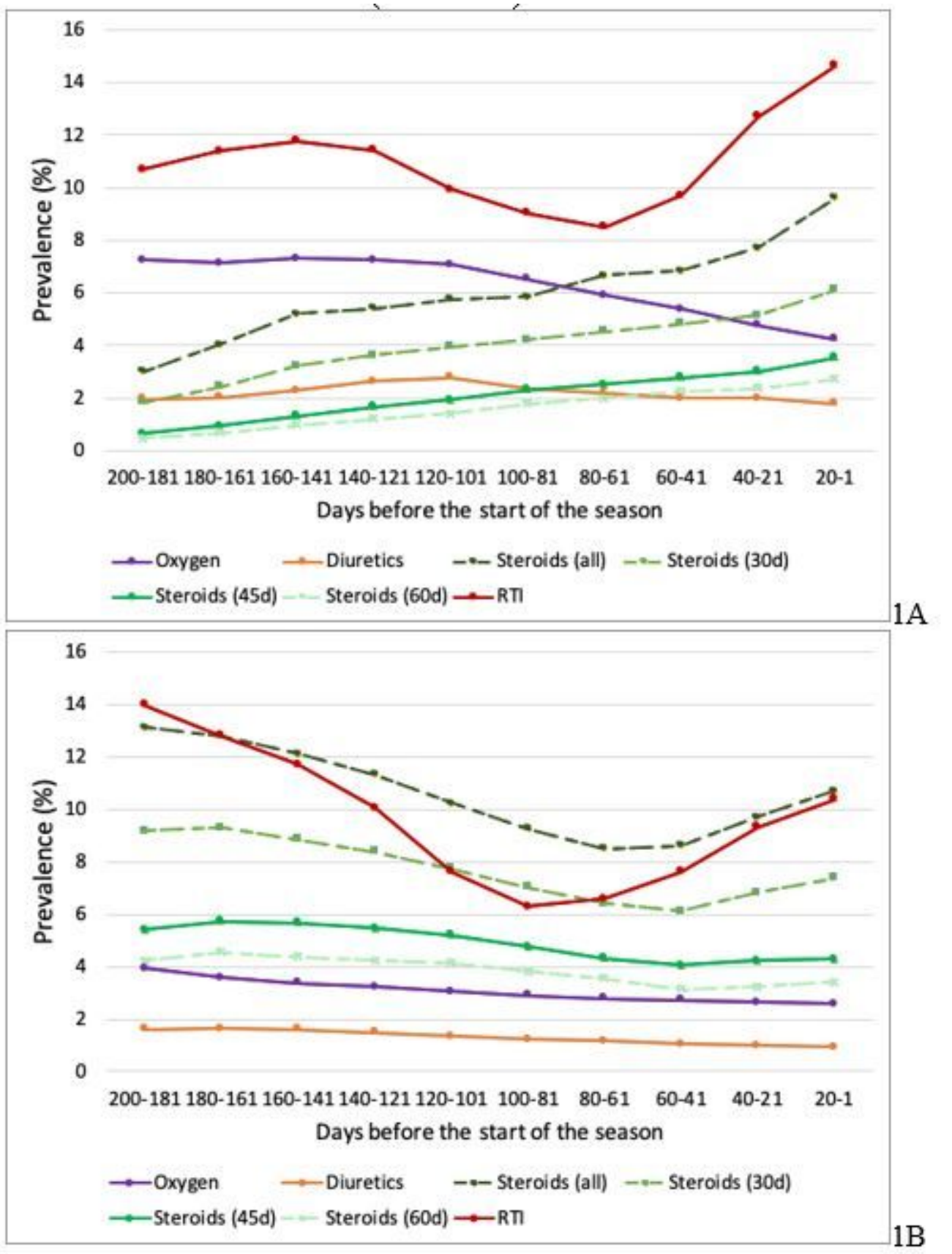

\section{Figure 1}

Prevalence of CLD treatment and RTI before the start of RSV season A) CLD infants between 200-365 days of age at the start of the season $(n=6,980)$ and B) CLD infants between 366-730 days of age at the start of the season $(n=12,046)$. Data source: MAX databases (1999-2010) linked to Florida and Texas Birth Certificate data. 